\title{
Reversible Cerebral Vasoconstriction Syndrome: A Commonly Missed Diagnosis in Acute Severe Headaches
}

\author{
Siriporn Hirunpat, MD., Nuttha Sanghan, MD., Adchara Wan-ai, MD., \\ Natee Ina, MD., Pornrujee Hirunpat, MD. \\ Department of Radiology, Faculty of Medicine, Prince of Songkla University \\ Hat-Yai, Songkhla, Thailand 90110
}

\begin{abstract}
Summary: In spite of the recent acceptance to be a common cause of acute severe headaches, reversible cerebral vasoconstriction syndrome (RCVS) is still commonly missed.

The syndrome is characterized by acute severe headaches due to the vasoconstriction of the cerebral arteries, which eventually resolve spontaneously. We present a reported case of RCVS with a typical magnetic resonance angiography (MRA) appearance and a review of the literature.
\end{abstract}

* Corresponding author: Hirunpat S, MD., Email: hirsirip@hotmail.com 


\section{Case report}

A 38-year-old man presented with the sudden onset of a severe headache, the feeling of almost syncope (presyncope) and dizziness. The patient was previously healthy and had no underlying disease. On clinical examination, the patient had full consciousness with a blood pressure of $130 / 80 \mathrm{~mm}$ $\mathrm{Hg}$. No evidence of neurological deficit was found and all basic investigations revealed normal results. He was sent for an MRI and MRA in order to search for the cause of his acute severe headache, such as ruptured aneurysm, and to exclude the possibility of minor stroke.

MRI and MRA studies were performed with a 3-Tesla imaging unit (Archieva-X series, Phillips Healthcare, Best, the Netherlands). The results were reported as normal by a radiologist. Therefore, chronic stress and migraine were diagnosed as the causes of his symptoms by the clinician.
Later consultation with a neuro-radiologist, multiple scatter foci of tiny high signal intensities on SET2w and FLAIR within the subcortical white matter of both frontal and left parietal lobes were detected (Fig.1). Although these lesions are commonly seen as part of normal aging, or due to underlying small vessel disease such as DM or HT, other causes, such as migraines, vascular dissection, anoxia or vasculopathy, should be of concern when detected in younger patients that do not have a definite underlying disease like in this case.

The MRA study of intracranial arteries was first performed using the 3-dimensional time- offlight (3D TOF) technique ( $F A=20^{\circ}, \mathrm{TR} \sim 25 \mathrm{msec}$, TE 3.45 msec, matrix size $500 \times 287$, FOV=139x139 $\mathrm{mm}$ ) and the post processing, by maximum intensity projection (MIP) technique, revealed segmental narrowing and dilatation along the wall of the $\mathrm{M} 2$ segment of the left middle cerebral artery (MCA)

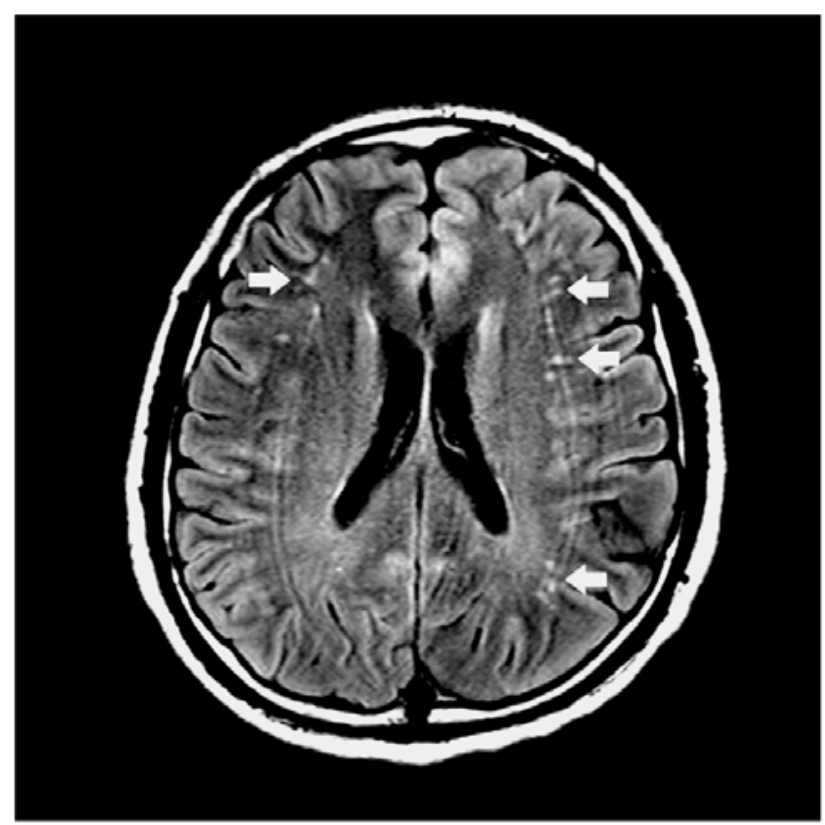

Fig 1. Axial FLAIR MR image revealed multiple scatter foci of tiny high signal intensities within the subcortical white matter of both frontal and left parietal lobes (arrows). 
May-August 2013, Volume XIX No.II

and the $\mathrm{A} 2$ segments of both anterior cerebral arteries (ACA), which resembled sausages on a string (Fig. 2).

With regard to the possibility of artifacts mimicking a lesion, on the following day the patient underwent a second MRA study which incorporated a number of MRA techniques, such as 3DTOF $\left(\mathrm{FA}=20^{\circ}, \mathrm{TR} \sim 25 \mathrm{msec}\right.$, TE $3.45 \mathrm{msec}$, matrix size $328 \times 572, F O V=152 \times 152 \mathrm{~mm}$ ), 3D PC (venc 45 $\mathrm{cm} / \mathrm{sec}, \mathrm{TR}=6.63 \mathrm{msec}, \mathrm{TE}=3.69 \mathrm{msec}$, matrix size $272 \times 272$, FOV=134×134 mm) and post contrast enhanced MRA ( $F A=30^{\circ}, \mathrm{TR}=4.86 \mathrm{msec}$, TE $1.67 \mathrm{msec}$, matrix size 250x400, FOV=200x200 mm). Persisting irregularities along the wall of the $\mathrm{M} 2$ segment of the left MCA and the A2 segments of both ACAs were noted in all the studies (Fig 3) and a nonspecific cause of vasculopathy was suggested.

Since no definite cause of vasculopathy or vasculitis could be found upon further investigations, the patient was diagnosed as a complicated migraine. Symptomatic treatments were provided. Follow-up MRA studies at about 6 weeks and the following year (Fig. 4a\&4b) revealed a full recovery of the vasoconstriction and RCVS was diagnosed. The patient also recently had a 3-year clinical follow-up. His severe headache has not returned and he is fine.

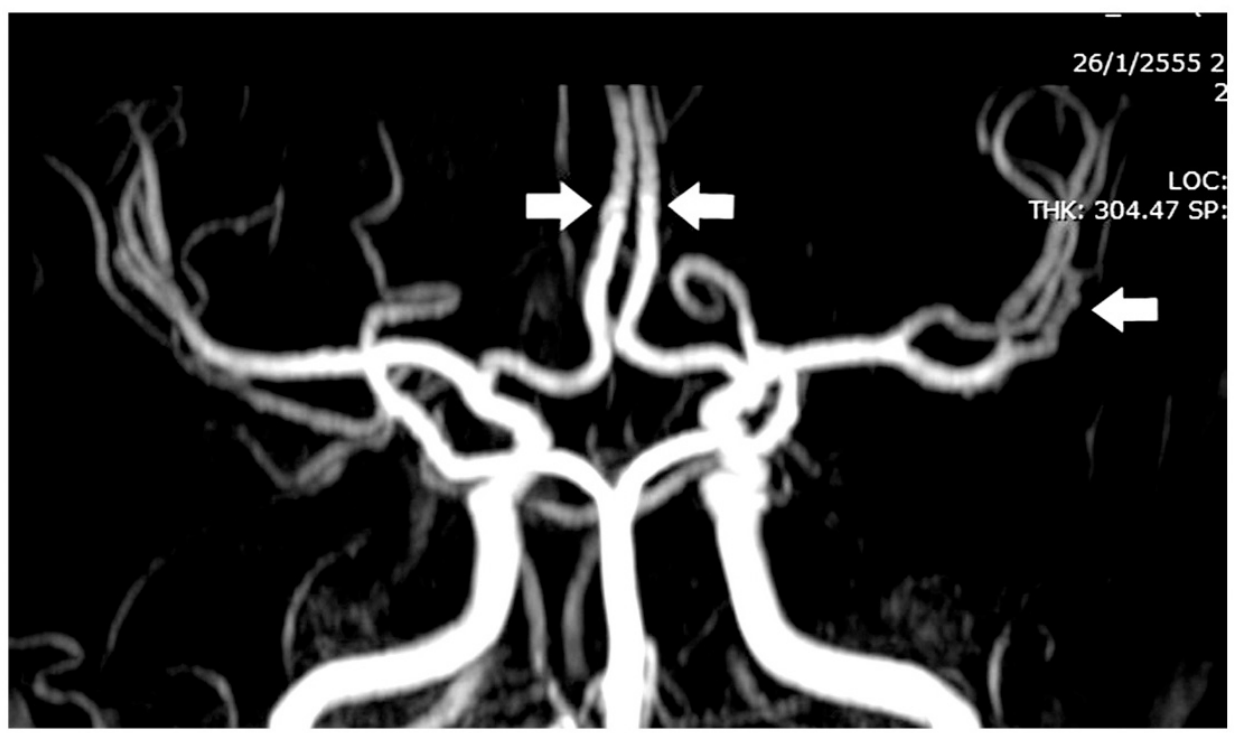

Fig 2. 3D TOF MRA (FA=20, TR $25 \mathrm{msec}$, TE $3.45 \mathrm{msec}$, matrix size 500x287, FOV $=139 \times 139 \mathrm{~mm}$ ) The post processing by MIP revealed segmental narrowing \& dilatation along the wall of the M2 segment of the left MCA and the A2 segments of both ACAs, like sausages on a string appearance ( arrows). 


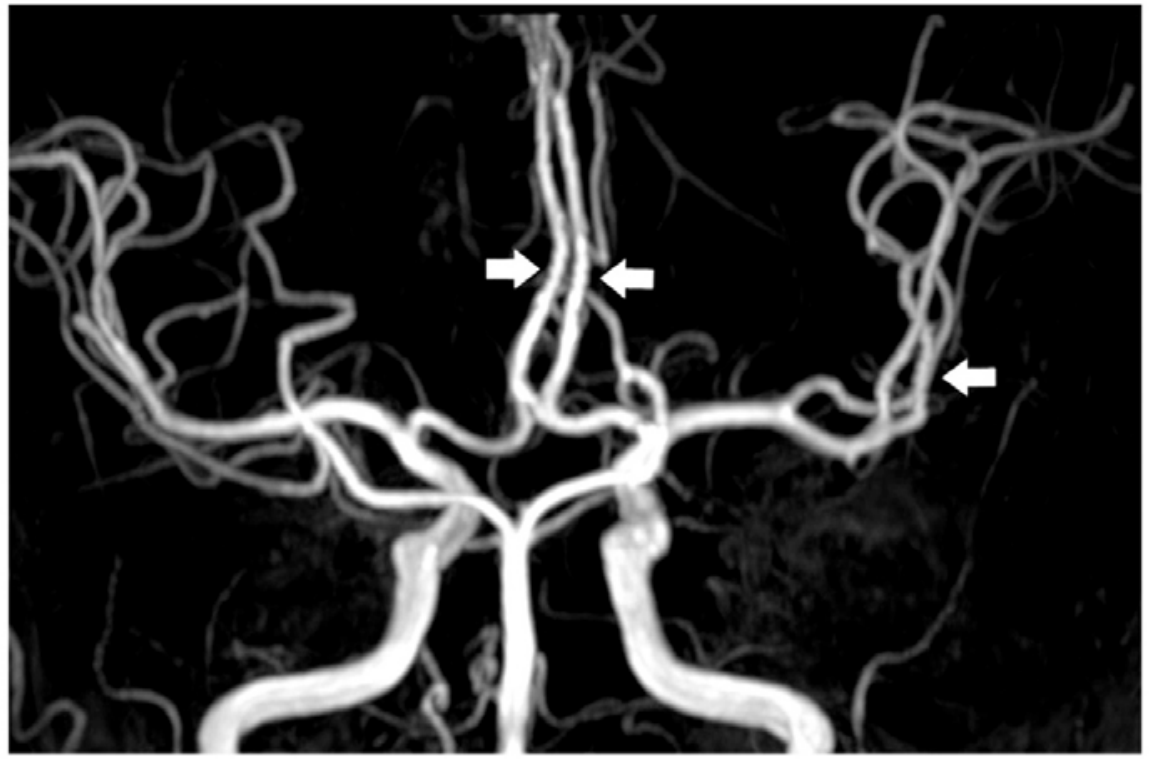

Fig 3. 3D TOF MRA (FA=20, TR $25 \mathrm{msec}$, TE $3.45 \mathrm{msec}$, matrix size $328 \times 572$, FOV $=152 \times 152 \mathrm{~mm}$ ) ) on the following day. Persisting irregularities along the wall of the $\mathrm{M} 2$ segment of the left MCA and the A2 segments of both ACAs were noted( arrows).

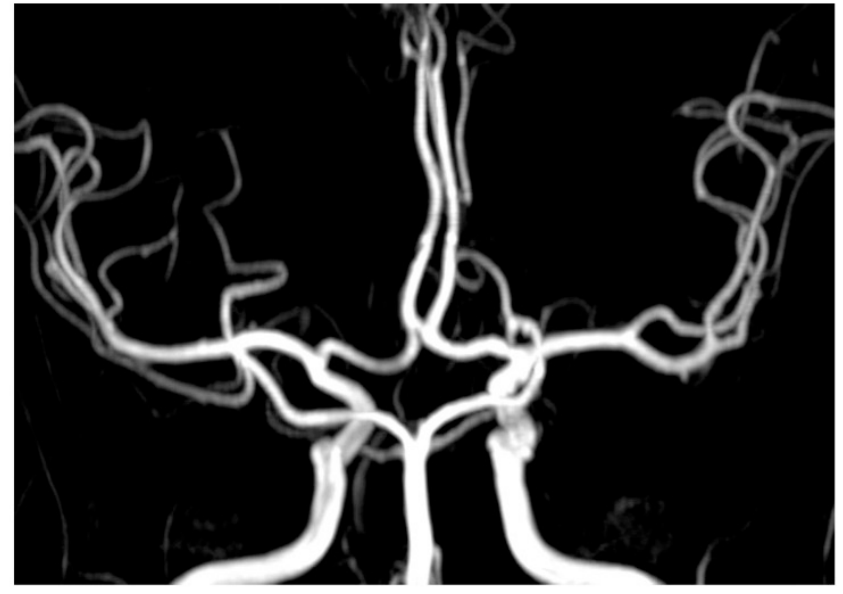

a.

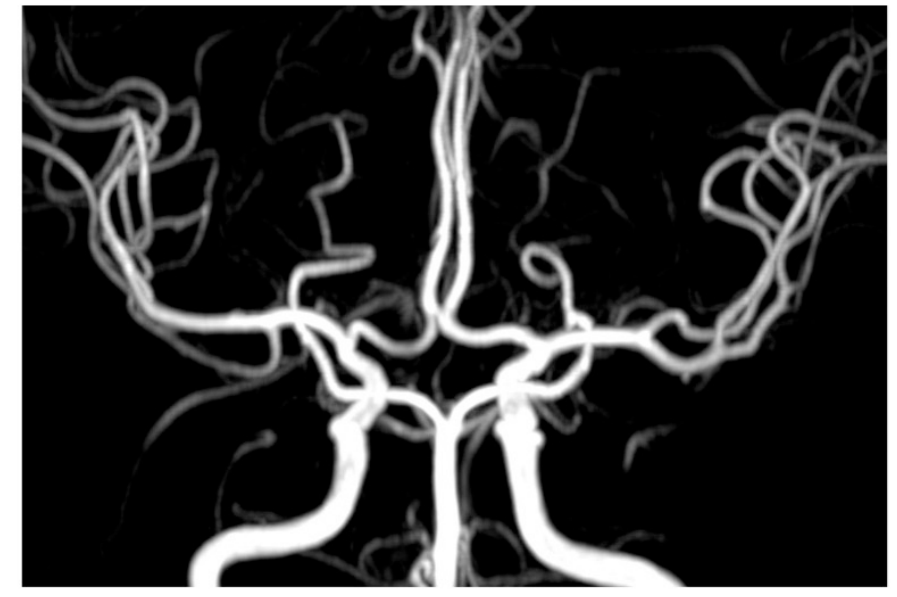

b.

Fig 4.3D TOF MRA at 6 weeks (a) and 1 year (b) revealed a full recovery of the vasoconstriction. 


\section{Discussion}

Reversible cerebral vasoconstriction syndrome (RCVS) is characterized by acute severe headache due to the constriction of the cerebral arteries which will eventually resolve spontaneously. It was first described by Call et al. in $1988^{(1)}$, followed by Calabrese et al. in 1993. ${ }^{(2)}$ The acute severe headache was often described as a "thunderclap in nature" because of the sudden excruciating pain reaching its maximal intensity within 1 minute, like a "clap of thunder" mimicking that of a ruptured aneurysm. Screaming, crying, agitation, confusion and collapse are also common. Associated with photophobia, nausea/vomiting and focal neurological deficit secondary to ischemia or hemorrhage can sometimes occur. Most thunderclap headaches are as short as a few minutes; however, some have been reported to last as long as several days. A single attack is possible, but usually patients have a mean of 4 attacks during 1-4 weeks. ${ }^{(3)}$

RCVS affects females more than males, with the variable frequency for females between $64-92 \%{ }^{(4)}$, and the mean age of onset is around 42 years. ${ }^{(5,6)}$

Diagnosis requires the demonstration of segmental narrowing and dilatation along the walls of the affected cerebral arteries, which resemble a "string of beads" or "sausages on a string" on an angiography, and complete resolution on a repeated angiography, within 3 months after onset. ${ }^{(7)}$

RCVS is a unifying term proposed in 2007 by Calabrese et al. ${ }^{(8)}$ which encompasses a group of recurrent headache syndromes, including CallFleming syndrome, benign angiopathy of the CNS, post-partum angiopathy, drug induced arteritis, migrainous vasospasm, migraine angiitis, primary (or idiopathic) thunderclap headache with reversible vasospasm, fatal vasospasm in migrainous infarc- tion, drug induced cerebral vasculopathy, and CNS pseudovasculitis. ${ }^{(7,9,10)}$ Since then, reports of the condition have been accumulating rapidly. In spite of the unknown exact incidence, it has been postulated that RCVS is under-recognized and is a common cause of acute severe headache..$^{(11-14)}$

RCVS can occur spontaneously (idiopathic) or be secondary to a precipitating factor. The proportion of spontaneous cases has varied depending on the studied populations, from $37 \%$ in a French study ${ }^{(5)}$ to $96 \%$ in a Taiwanese cohort. ${ }^{(9)}$ Precipitating factors include postpartum state, migraine history and exposure to vasoactive substances, such as cannabis, cocaine, ecstasy, amphetamines, binge drinking, selective serotonin reuptake inhibitors, triptans, nasal decongestants, ergotamine tartrate, LSD (lysergic acid diethylamide), methergine, bromocriptine, nicotine patches, ginseng and caffeine ${ }^{(7,15,16)}$ Other causes such as hypercalcemia, pheochromocytoma, exertion, and sexual activity also have been described to be related with this syndrome. ${ }^{(7,13,17)}$ The syndrome may occur after a few days of exposure or after several months of regular or irregular exposure in normal or excessive doses. Post-partum RCVS has been described to start in two-thirds of cases during the first week after delivery, usually after a normal pregnancy. In $50-70 \%$ of cases it is associated with the intake of vasoconstrictors, mostly ergots used to treat post-partum hemorrhage or to inhibit lactation. ${ }^{(7)}$

Despite recently accepted to be a common cause of acute severe headaches, RCVS is still commonly missed due to its recent description in literature. Furthermore, the degree of vasoconstriction may be too subtle to be definitely detected, or the detected lesion may be missed classified as vasculitis or other causes of vasculopathy. 
RCVS is likely to be one of the most common vasculopathies that is confused with vasculitis. Vasculopathy is any disorder of blood vessels while vasculitis implies inflammation of blood vessels. Distinguishing RCVS from vasculitis seen in primary angiitis of the CNS (PACNS), which often requires brain biopsy for definite diagnosis, is very important for determining the appropriate treatment. Treatment for PACNS is much more aggressive, often including steroids and immunosuppressive agents with substantial adverse effects. By contrast with RCVS, PACNS usually has an insidious onset. Headaches are frequent but not of the thunderclap type and are followed by a stepwise deterioration. MRI scans are abnormal in most cases and show several small deep or superficial infarcts of different ages with or without white matter abnormalities. CSF analysis also shows an inflammatory reaction. Angiography is frequently normal in PACNS, whereas, by definition, it is always abnormal in RCVS (except when done early). ${ }^{(3)}$ Some features suggest PACNS, irregular eccentric and asymmetrical narrowing or several occlusions on angiograms and contrast enhancement of the vessel wall in MRI. ${ }^{(18,19)}$ When the clinicians remain unsure of the diagnosis, waiting for a few days might be best. RCVS should stabilize, improve quickly and resolution of vasoconstriction, whereas arterial irregularities in PACNS do not improve rapidly. ${ }^{(6)}$ Response to intra-arterial nimodipine has been proposed as a differential diagnosis test that remains to be validated; the drug immediately normalized arterial abnormalities in a few cases of RCVS, but is not expected to change the lesions in PACNS. ${ }^{(20,21)}$

The diagnosis of RCVS does not require aggressive brain biopsy but can be suggested by the following triad;
1. Appropriate clinical history (acute severe or thunderclap headache)

2. Eventual resolution of angiographic vasoconstriction (within 3 months)

3. Absence of inflammatory/infection etiology.

The sensitivity and specificity of various imaging modalities for RCVS have not been established but cerebral angiogram is generally accepted to be the gold standard for the diagnosis. Since angiography is not a convenient tool for frequent follow-up, CTA or

MRA could be good alternative options. CTA and MRA also have been reported to be valuable tools for clinical evaluation and risk assessment in patients with RCVS. ${ }^{(17,22,23)}$

The large amount of bony artifacts from the skull base interfering with the arteries around the Circle of Willis causes the more popularly used MRA than CTA in our institution. Furthermore, MRI, which is generally more sensitive to brain lesions than CT, can be performed in the same setting.

MRI in RCVS can be normal, but may also show small ischemic/infarcts lesions, especially in the posterior circulation and watershed areas. ${ }^{(3,6,15)}$ The incidences of infarcts vary between $4-39 \%$. $^{(3,6,24)}$ The MRI brain scan of this 38-year-old patient without underlying disease revealed multiple scatter foci of tiny high signal intensities on T2w and FLAIR within the watershed ischemic distributions of the subcortical white matter of both frontal and left parietal lobes. Although similar multiple scatter foci of high signal intensities are commonly seen in the elderly, as part of normal aging, other causes of small vessel disease, such as DM, HT, migraine, vascular dissection, anoxia or other non-specific causes of vasculopathy, should be of concern in younger patients, like in this case. 
Vasoconstriction in RCVS is most common in large- and medium-sized vessels. ${ }^{(25)}$ Although an MRA can demonstrate vasoconstriction in the proximal parts of the cerebral arteries, a normal MRA does not rule out the diagnosis. In a series of 44 RCVS patients, 6 had a normal MRA while with the cerebral angiography there were signs of vasoconstriction. ${ }^{(26)}$ Vasoconstriction can persist for weeks after the resolution of the headaches ${ }^{(3)}$ but resolution of vasoconstriction is expected in all patients at repeat angiography within 3 months. In our patient, complete resolution of vasoconstriction was seen at 6 weeks; the patient recovered fully without any specific treatment. Follow-up MRA at 1 year, which was conducted in order to write this report, revealed no evidence of vasoconstriction.

The exact cause of RCVS is still unknown. The sympathetic nervous system may play a role in the pathogenesis of this headache since this headache has been reported to be associated with multiple factors elevating the sympathetic tone, such as adrenergic drugs and physical stress. ${ }^{(27)}$ Furthermore, proximal parts of the intracranial vessels are also strongly innervated by sympathetic afferents, which modulate vascular tone.

RCVS and posterior reversible encephalopathy syndrome (PRES) are frequently associated. Reversible brain edema occurs about $8-38 \%$ of RCVS. ${ }^{(3,16,28)}$ Moreover, a multifocal cerebral vasoconstriction has been noted in more than $85 \%$ of patients with PRES whenever the investigation included angiography. This vasoconstriction was shown to be reversible on follow-up MRA. ${ }^{(3)}$ PRES can complicate toxaemia of pregnancy, immunosuppressive treatment after transplantation, chemotherapy, autoimmune diseases, hypertension and septic shock, all of which are associated with endothelial damage or activation. It was thought to be caused by severe hypertension, leading to altered cerebral autoregulation with hyperperfusion and vasogenic edema. However, a quarter of the patients with PRES are normotensive. A more recent view is that endothelial dysfunction of any cause can affect the regulation of cerebral arterial tone and trigger vasoconstriction with subsequent hypoperfusion, and breakdown of the blood-brain barrier and vasogenic edema. $^{(3,7,29)}$

Intracranial hemorrhage, especially cortical subarachnoid hemorrhage (SAH), has been reported to accompany RCVS. ${ }^{(26,28,30-32)} \mathrm{Up}$ to $34 \%$ of patients with RCVS developed intracranial hemorrhage ${ }^{(28)}$ and the most common intracranial hemorrhage was tiny localized cortical subarachnoid hemorrhage, unilateral or bilateral, which was seen as much as $22-50 \%$ in patients with RCVS. ${ }^{(28,31)}$

Vasoconstriction as a result of subarachnoid hemorrhage is well-known, but vasoconstriction as the cause of subarachnoid hemorrhage is rather difficult to believe without question. Ducros et al. hypothesized an interesting concept, in an opposite way to the others, that arterial abnormalities first involve small distal arteries and then progress toward medium-and large-sized vessels, which could explain the high rate of normal early angiogram( up to $33 \%)$. Moreover, marked vasoconstriction could persist for weeks after headache resolution, suggesting that vasoconstriction was not the direct cause of the headache.

Segmental vasodilatation is likely to play an important role in the initial stage of RCVS, triggering thunderclap headaches by abruptly stretching the vessel walls and causing hemorrhage by small vessel rupture or reperfusion injury. In the second stage, vasoconstriction of the second and first 
segments of the major cerebral arteries becomes the major problem causing ischemia or mainly watershed infarction. ${ }^{(3,23)}$

RCVS also has been recently included as a possible cause of spontaneous subarachnoid hemorrhage, especially for tiny localized cortical subarachnoid hemorrhage situated far from the Circle of Willis, which is usually easy to distinguish from aneurysmal subarachnoid hemorrhage. Besides RCVS, small convexity bleeding can occur in amyloid angiopathy.

A retrospective study suggested that RCVS appears to be a common cause of convexity subarachnoid hemorrhage in patients aged 60 years or younger, whereas amyloid angiopathy is frequent in those older than 60 years. ${ }^{(33)}$

In spite of possible serious complications, the rate of permanent neurological disability is low. ${ }^{(14)}$ Most patients do well and recover completely without neurological sequelae, especially in the spontaneous cases. ${ }^{(13,14,24)}$ Migraine history, post-partum status and cannabis use have been observed to be more related with stroke and poor outcome. ${ }^{(13,23)}$ Recurrence of the syndrome is possible. The rate is unknown but is probably low. ${ }^{(3)}$

There is no established therapy in RCVS. Good outcomes have been reported with symptomatic treatment, the discontinuation of any possibly related substances or triggers, and rest for a few days to a few weeks according to the severity of the headaches. ${ }^{(734)}$ Benzodiazepine to relieve anxiety, which is common and could be an aggravating factor, is recommended in some institutions. ${ }^{(3)}$ In most cases, headaches and angiographic abnormalities resolve within days or weeks. Because of the similarities with post subarachnoid hemorrhagerelated vasospasm, calcium channel blockers are widely used in the treatment of RCVS. Nimodipine has been shown to terminate the headache within 48 hours in $63-84 \%$ of patients ${ }^{(3,5,9,14,35,36)}$, in spite of no outcome benefit over symptomatic alone in a large case series report. ${ }^{(6)}$ Short-term high dose glucocorticoids had been recommended because of radiological similarities with vasculitic disorders and experimental evidence that glucocorticoids reduce vasoconstriction. ${ }^{(18,37)}$ However, they have recently been shown to be an independent predictor of poor outcome and should be avoided. ${ }^{(6,14)}$ The use of intravenous magnesium sulfate in patients with post-partum RCVS also has been reported to produce good results. ${ }^{(24)}$

\section{Conclusions}

RCVS is still under-recognized. The primary diagnostic dilemma is distinguishing RCVS from primary CNS arteritis and RCVS should be included in the differential diagnosis as a possible cause of tiny cortical subarachnoid hemorrhage. Complete resolution of vasoconstriction of cerebral arteries in 3 months without evidence of inflammatory/infection etiology should suggest the diagnosis.

\section{Acknowledgments}

Special thanks to Mr.Trevor T. Pearson for proof reading and editing assistance.

\section{References}

1. Call GK, Fleming MC, Sealfon S, Levine H, Kistler JP, Fisher CM. Reversible cerebral segmental vasoconstriction. Stroke 1988;19:1159-70.

2. Calabrese LH, Gragg LA, Furlan AJ. Benign angiopathy: a distinct subset of angiographically defined primary angiitis of the central nervous system. J Rheumatol 1993;20: 2046-50. 
3. Ducros A. Reversible cerebral vasoconstriction syndrome. Lancet Neurol 2012;11:906-917.

4. Velez A, McKinney J. Reversible Cerebral Vasoconstriction Syndrome: A Review of Recent Research. Current Neurology and Neuroscience Reports 2012;13:1-5.

5. Ducros A, Boukobza M, Porcher R, Sarov M, Valade D, Bousser M. The clinical and radiological spectrum of reversible cerebral vasoconstriction syndrome: a prospective series of 67 patients. Brain 2007;130:3091-101.

6. Singhal A, Hajj-Ali R, Topcuoglu M, Fok J, Bena J, Yang $D$, et al. Reversible cerebral vasoconstriction syndromes: analysis of 139 cases. Arch Neurol 2011;68:1005-12.

7. Ducros A, Bousser MG. Reversible cerebral vasoconstriction syndrome. Pract Neurol 2009;9:256-67.

8. Calabrese LH, Dodick DW, Schwedt TJ, Singhal AB. Narrative Review: Reversible Cerebral Vasoconstriction Syndromes. Annals of Internal Medicine 2007;146:34-44.

9. Chen SP, Fuh JL, Lirng JF, Chang FC, Wang SJ. Recurrent primary thunderclap headache and benign CNS angiopathy: spectra of the same disorder? Neurology 2006;67:2164-9.

10. Chen SP, Fuh JL, Wang SJ. Reversible cerebral vasoconstriction syndrome: current and future perspectives. Expert Rev Neurother 2011;11:1265-76.

11. Chen S, Fuh J, Wang S. Reversible cerebral vasoconstriction syndrome: an under-recognized clinical emergency. Ther Adv Neurol Disord 2010;3:161-71.

12. Headache Classification Committee of the International Headache S. The International Classification of Headache Disorders, $3^{\text {rd }}$ edition (beta version). Cephalalgia 2013;33:629-808.

13. Cheng Y-C, Kuo K-H, Lai T-H. A common cause of sudden and thunderclap headaches: reversible cerebral vasoconstriction syndrome. J Headache Pain 2014;15:13.

14. Tan LH, Flower O. Reversible Cerebral Vasoconstriction Syndrome: An Important Cause of Acute Severe Headache. Emergency Medicine International 2012;2012:8.

15. Koopman K, Teune LK, ter Laan M, Uyttenboogaart M, Vroomen PC, De Keyser J, et al. An often unrecognized cause of thunderclap headache: reversible cerebral vasoconstriction syndrome. J Headache Pain 2008;9:389-91.

16. Hijikata $\mathrm{Y}$, Watanabe $\mathrm{H}$, Ito $\mathrm{M}$, Yoneyama $\mathrm{N}$, Atsuta $\mathrm{N}$, Sobue G. Reversible cerebral vasoconstriction syndrome
(RCVS) triggered by nicotine patches. Rinsho Shinkeigaku 2013;53:721-3.

17. Marder CP, Donohue MM, Weinstein JR, Fink KR. Multimodal Imaging of Reversible Cerebral Vasoconstriction Syndrome: A Series of 6 Cases. American Journal of Neuroradiology 2012;33:1403-11.

18. Hajj-Ali RA, Furlan A, Abou-Chebel A, Calabrese LH. Benign angiopathy of the central nervous system: Cohort of 16 patients with clinical course and long-term followup. Arthritis Care \& Research 2002;47:662-9.

19. Mandell DM, Matouk CC, Farb RI, Krings T, Agid R, terBrugge $\mathrm{K}$, et al. Vessel Wall MRI to Differentiate Between Reversible Cerebral Vasoconstriction Syndrome and Central Nervous System Vasculitis: Preliminary Results. Stroke 2012;43:860-2.

20. Elstner M, Linn J, Muller-Schunk S, Straube A. Reversible cerebral vasoconstriction syndrome: a complicated clinical course treated with intra-arterial application of nimodipine. Cephalalgia 2009;29:677-82.

21. Linn J, Fesl G, Ottomeyer C, Straube A, Dichgans M, Bruckmann $\mathrm{H}$, et al. Intra-arterial application of nimodipine in reversible cerebral vasoconstriction syndrome: a diagnostic tool in select cases? Cephalalgia 2011;31: 1074-81.

22. Chen SP, Fuh JL, Wang SJ, Chang FC, Lirng JF, Fang $Y C$, et al. Magnetic resonance angiography in reversible cerebral vasoconstriction syndromes. Ann Neurol 2010;67: 648-56.

23. Marder CP, Donohue MM, Weinstein JR, Fink KR. Multimodal imaging of reversible cerebral vasoconstriction syndrome: a series of 6 cases. AJNR Am J Neuroradiol 2012;33:1403-10.

24. Sattar A, Manousakis G, Jensen MB. Systematic review of reversible cerebral vasoconstriction syndrome. Expert Rev Cardiovasc Ther 2010;8:1417-21.

25. Saini M, Jeerakathil T, Butcher K. Reversible cerebral vasoconstriction syndrome. Neurol India 2009;57:63-5.

26. Ducros A, Boukobza M, Porcher R, Sarov M, Valade D, Bousser MG. The clinical and radiological spectrum of reversible cerebral vasoconstriction syndrome. A prospective series of 67 patients. Brain 2007:130:3091-101.

27. Lu SR, Liao YC, Fuh JL, Lirng JF, Wang SJ. Nimodipine for treatment of primary thunderclap headache. Neurology 2004;62:1414-6. 
28. Ducros A, Fiedler U, Porcher R, Boukobza M, Stapf C, Bousser MG. Hemorrhagic manifestations of reversible cerebral vasoconstriction syndrome: frequency, features, and risk factors. Stroke 2010;41:2505-11.

29. Bartynski WS, Boardman JF. Catheter angiography, MR angiography, and MR perfusion in posterior reversible encephalopathy syndrome. AJNR Am J Neuroradiol 2008;29:447-55.

30. Edlow B, Kasner S, Hurst R, Weigele J, Levine J. Reversible Cerebral Vasoconstriction Syndrome Associated with Subarachnoid Hemorrhage. Neurocritical Care 2007;7: 203-10.

31. Muehlschlegel S, Kursun O, Topcuoglu MA, Fok J, Singhal AB. Dlfferentiating reversible cerebral vasoconstriction syndrome with subarachnoid hemorrhage from other causes of subarachnoid hemorrhage. JAMA Neurology 2013;70:1254-60.

32. Ducros A, Boukobza M, Porcher R, Sarov M, Valade D, Bousser M-G. The clinical and radiological spectrum of reversible cerebral vasoconstriction syndrome. A prospective series of 67 patients. Brain 2007;130:3091-101.

33. Kumar S, Goddeau RP, Selim MH, Thomas A, Schlaug G, Alhazzani A, et al. Atraumatic convexal subarachnoid hemorrhage: Clinical presentation, imaging patterns, and etiologies. Neurology 2010;74:893-9.

34. Singhal A, Bernstein R. Postpartum angiopathy and other cerebral vasoconstriction syndromes. Neurocritical Care 2005;3:91-7.

35. Dodick D. Reversible Segmental Cerebral Vasoconstriction (Call-Fleming Syndrome): the Role of Calcium Antagonists. Cephalalgia 2003;23:163-5.

36. Lu S-R, Liao Y-C, Fuh J-L, Lirng J-F, Wang S-J. Nimodipine for treatment of primary thunderclap headache. Neurology 2004;62:1414-6.

37. Werring DJ. Reversible Cerebral Vasoconstriction Syndrome and Intracranial Hemorrhage: Some Answers, Many Questions. Stroke 2010;41:2455-6. 